parameter, but this term can be eliminated from the energy level analysis by the proper choice of coordinate axes. This involves a rotation about the $c$ axis and results in no loss of generality in the energy level analysis. Discussion of this point can be found in J. L. Prather, Natl. Bur. Std. (U.S.) Monograph 19 (1961), or Appendix A of Ref. 39.

31 B. G. Wybourne, Spectroscopic Properties of Rare Earths (Interscience, New York, 1965).

${ }^{32}$ S. S. Bishton and D. J. Newman, J. Phys. C 3, 1753 (1970).

${ }^{33} \mathrm{~K}$. Rajnak, J. Chem. Phys. 43, 847 (1965). A typographical error was found in the eigenvector for the $J=11 / 2$ level at $10000 \mathrm{~cm}^{-1}$ by checking the orthogonality of the eigenvectors. The sign of the ${ }^{2} H(21)$ component should be + .

${ }_{34}$ J. C. Eisenstein, J. Chem. Phys. 39, 2128 (1963).

${ }^{35}$ K. Rajnak and B. G. Wybourne, Phys. Rev. 132, 280 (1963).

${ }^{36} \mathrm{~K}$. Rajnak and B. G. Wybourne, Phys. Rev. 134, A596 (1964).
${ }^{37}$ B. R. Judd, Proc. Phys. Soc. 74, 330 (1959).

${ }^{38}$ C. W. Nielson and G. F. Koster, Spectroscopic Coefficients of the $p^{n}, d^{n}$, and $f^{n}$ Configurations (MIT Press, Cambridge, 1963).

${ }^{39}$ R. L. Cone, Ph.D. thesis, Yale Univ., 1971, Appendix D, University Microfilm 71-28151.

${ }^{40}$ For a Fortran IV listing of the program, see Ref. 39, Appendix

${ }^{41}$ J. C. Morrison, P. R. Fields, and W. T. Carnall, Phys. Rev. B 2, 3526 (1970).

42 W. P. Wolf, Int. J. Quantum Chem. Symp. 5, 397 (1971).

${ }^{43}$ G. F. Koster, J. O. Dimmock, R. G. Wheeler, and Hermann Statz, Properties of the Thirty-two Point Groups (MIT Press, Cambridge, 1963).

${ }^{44}$ To determine the absolute sign experimentally, circularly polarized radiation must be used.

${ }^{45}$. Due to the crystal structure and the Ising nature of the $\mathrm{Tb}^{3+}$ ground state, the perpendicular components of $\mathbf{u}$ are not involved.

\title{
Theory of Semiclassical Transition Probabilities for Inelastic and Reactive Collisions. V. Uniform Approximation in Multidimensional Systems*
}

\author{
R. A. Marcus \\ Department of Chemistry, University of Illinois, Urbana, Illinois 61801
}

(Received 14 April 1972)

\begin{abstract}
An integral semiclassical expression for the $S$ matrix of inelastic and reactive collisions was formulated earlier in this series. In the present paper a uniform approximation for the expression is derived for the case of multidimensional systems. The method is an extension of that employed in Part II for the case of one internal coordinate. The final result, Eq. (2), is highly symmetrical, thus making some of its properties immediately clear.
\end{abstract}

\section{INTRODUCTION}

In semiclassical theory for inelastic or reactive collisions, ${ }^{1-3}$ an inelastic transition from state $n$ to state $m$ is associated with a particular classical trajectory. The latter is one for which the (continuously varying) "quantum number" $\bar{n}$ equals $n$ before collision and $m$ after it. Such a trajectory corresponds to a point of stationary phase of the phase term in the exponent of the integrand in a semiclassical expression for the $S$-matrix element $S_{m n}{ }^{1,2}$ Frequently, several such trajectories contribute and give rise to interference phenomena for the transition, and for scattering. Often the stationary phase value of the integral suffices, and one then has

$$
S_{m n}=\sum_{j} I_{j}
$$

where the sum is over all stationary phase points $j$ of the integrand and where $I_{j}$ is the contribution to $S_{m n}$ from the $j$ th point.

When two or more of the stationary phase points are close together, the effects of this proximity must be included (the "uniform approximation"). The case of one dimension was treated in Part $\mathrm{II}^{\mathrm{1b}}$ by adapting a method of Ursell, Chester, and Friedman. This method, which has also been used for obtaining a uniform approximation for the rainbow and glory effects in elastic scattering, ${ }^{5}$ involves mapping the exponent in the integrand onto a cubic polynomial and mapping the pre-exponential factor onto a power series. ${ }^{6}$ One then obtains a uniform asymptotic expansion. The case of one and two dimensions has been also treated more intuitively, ${ }^{7}$ obtaining the latter by interpolating from various limiting cases.

Apart from the collision of a rigid rotor and an atom, inelastic or reactive molecular collisions involve at least three internal coordinates in the integral expression for $S_{m n}$. In the present paper we treat the multidimensional case by extending the method of Part II. We obtain

$$
S_{m n}=\sum_{j} I_{j} B i^{(+)}\left(-\zeta_{j j_{1}}\right) \cdots B i^{(+)}\left(-\zeta_{j j_{N}}\right),
$$

where the sum is over all stationary phase points $j$, the latter's neighbors being $j_{1} \cdots j_{N}$. The points $j$ are put into $1: 1$ correspondence with the corners of a hypercube and $j_{1}, \cdots, j_{N}$ are at the corners adjacent to $j$. The $B i^{(+)}$'s are certain combinations of the Airy integral and its derivative. The magnitude of $B i^{(+)}$ is usually close to unity and its phase rapidly decreases toward zero as $\zeta$ increases (as in Table I, given later). The $\zeta$ 's are related to phase integral differences between adjacent points $j$ and $j_{K}$. 


\section{EVALUATION OF INTEGRAL}

We begin with the case of two dimensions, the integral $S_{m n}$ being a particular example of an integral, $I,{ }^{8}$

$$
I=\iint g(x, y)[\exp i f(x, y)] d x d y .
$$

The evaluation reduces to that of one-dimensional integrals when one can separate variables, i.e., when $f(x, y)=f(x)+f(y)$ and $g(x, y)=g(x) g(y)$. However, such an approximation cannot be made in general. A typical situation is given schematically in Fig. 1, where the points of stationary phase are indicated as $P_{1}, P_{2}, P_{3}$, and $P_{4}$, there frequently being four points of stationary phase. Sometimes, as one sees by symmetry arguments, ${ }^{1 \mathrm{a}}$ there may be more, but the evaluation of the integral for $S_{m n}$ can then be reduced by symmetry to the evaluation of one with this smaller number of points. ${ }^{\text {a }}$ Sometimes, because of an unusually complicated (highly nonsinusoidal) phase integral, there may again be more points. In the separable case $P_{1}$ to $P_{4}$ are at the corners of a rectangle in this $x y$ plane.

We introduce in Fig. 1 curvilinear coordinates $u$ and $v$, and suppose the mapping $(x, y) \rightarrow(u, v)$ to be one to one and regular. The $u$ curves are chosen such that along them one may map the phase $f$ in Eq. (3) onto a cubic expression,

$$
f(x, y)=\frac{1}{3} u^{3}-\zeta u+A \quad \text { (along } u \text { curves). }
$$

Here, $\zeta$ and $A$ are constant along such a curve but their values can vary from member to member of this family of $u$ curves. Two $u$ curves are $P_{1} P_{4}$ and $P_{2} P_{3}$ in Fig. 1. $\zeta$ and $A$ are real, when (4) is valid over a sufficiently large region, since $f$ is real for real $x$ and $y$ and hence for real $u$.

The points of stationary phase, $P_{1}, P_{2}, P_{3}$, and $P_{4}$, are those for which

$$
\partial f / \partial x=\partial f / \partial y=0
$$

and thereby for which, along a $u$ curve

$$
d f / d u=0 \text {. }
$$

There are two solutions to (4) and (6), occurring at points which will be designated $S$ and $S^{\prime}$ :

$$
\begin{array}{ll}
u=-\zeta_{S S^{\prime}}{ }^{1 / 2} & \text { at } S \\
u=+\zeta_{S S^{\prime}}{ }^{1 / 2} & \text { at } S^{\prime},
\end{array}
$$

where $\zeta_{S S^{\prime}}$ is the $\zeta$ for the $u$ curve joining $S$ and $S^{\prime}$ (Fig. 1). The set of points $S$ is the curve $P_{1} P_{2}$ and that of $S^{\prime}$ is curve $P_{4} P_{3}$ there. Equations (4) and (7) yield the values $f(x, y)$ at $S$ and $S^{\prime}, f_{S}$ and $f_{S^{\prime}}$ :

$$
f_{S}=\frac{2}{3} \zeta_{S S^{\prime}}{ }^{3 / 2}+A_{S S^{\prime}}, \quad f_{S^{\prime}}=-\frac{2}{3} \zeta_{S S^{\prime}}{ }^{3 / 2}+A_{S S^{\prime}}
$$

and so

$$
f_{S}-f_{S^{\prime}}=\frac{4}{3} \zeta_{S S^{\prime}} 3 / 2, \quad f_{S}+f_{S^{\prime}}=2 A_{S S^{\prime}} .
$$

According as the curve $S S^{\prime}$ is taken to be $P_{1} P_{4}$ or $P_{2} P_{3}$, the corresponding $\zeta_{\mathrm{SS}^{\prime}}$ is designated as $\zeta_{14}$ or $\zeta_{23}$, respectively.

We introduce a coordinate $v$ along curves $P_{1} P_{2}$ and $P_{4} P_{3}$, such that

$$
f(x, y)=\frac{1}{3} v^{3}-\eta v+B \quad \text { (along a } v \text { curve). }
$$

$\eta$ and $B$ depend on the particular $v$ curve, e.g., $P_{1} P_{2}$ or $P_{4} P_{3}$, and are real. The stationary phase condition (5) implies that, along a $v$ curve,

$$
d f / d v=0
$$

at a stationary phase point, thus yielding solutions

$$
\begin{array}{ll}
v=-\eta_{Q Q^{\prime}}{ }^{1 / 2} & \text { at } Q \\
v=+\eta_{Q Q^{\prime}}{ }^{1 / 2} & \text { at } Q^{\prime},
\end{array}
$$

where $Q$ is $P_{1}$ or $P_{4}$ in Fig. 1 and $Q^{\prime}$ is $P_{2}$ or $P_{3}$. Curves $P_{1} P_{2}$ and $P_{4} P_{3}$ are members of a family of $v$ curves, as in Fig. 1.

Equations (9) and (11) applied to curves $P_{1} P_{2}$ and $P_{4} P_{3}$ lead to

$$
\begin{aligned}
& \frac{4}{3} \eta_{12}{ }^{3 / 2}=f_{1}-f_{2} \\
& \frac{4}{3} \eta_{43}{ }^{3 / 2}=f_{4}-f_{3} .
\end{aligned}
$$

From the foregoing it follows that

$$
\begin{array}{ll}
P_{1}\left(-\zeta_{14^{1 / 2}},-\eta_{12}{ }^{1 / 2}\right), & P_{2}\left(-\zeta_{23^{1 / 2}},+\eta_{12}^{1 / 2}\right), \\
P_{3}\left(+\zeta_{23^{1 / 2}},+\eta_{43^{1 / 2}}\right), & P_{4}\left(+\zeta_{14^{1 / 2}},-\eta_{43}^{1 / 2}\right) .
\end{array}
$$

Of these four solutions to (5) there may be four real, or some real and some complex-valued, or four complexvalued solutions. The case of one or more real ones yields a classically accessible transition while that of four complex ones yields a classically inaccessible one. ${ }^{1}$

The quantities $\zeta, A, \eta$, and $B$ have a useful property employed later: differentiation of (8b) with respect to $v$, and application of (10) when $S$ and $S^{\prime}$ are the stationary phase points $P_{1}$ and $P_{4}$ or $P_{2}$ and $P_{3}$, shows that along a $v$ curve

$$
d \zeta_{S^{\prime}} / d v=0 \quad \text { (at a stationary phase point) }
$$

and that $d A_{S S^{\circ}} / d v$ vanishes there also. Similarly using (8) but with $S, S^{\prime}, \zeta$, and $A$ replaced by $Q, Q^{\prime}, \eta$, and $B$, differentiating it with respect to $u$ and applying (6) shows that along a $u$ curve

$$
d \eta_{Q Q^{\prime}} / d u=0 \quad \text { (at a stationary phase point) }
$$

and that $d B_{Q Q^{\prime}} / d u$ also vanishes there.

The integral in Eq. (1) can be written as

$$
I=\iint g J\left[\exp i\left(\frac{1}{3} u^{3}-\zeta u+A\right)\right] d u d v,
$$

where $J$ denotes the actual (rather than absolute) value of the Jacobian for the transformation $(u, v) \rightarrow$ $(x, y)$. (This is an important point, partly because one is working with complex variables $x$ and $y$ and their 
functions.)

$$
J=\left(\begin{array}{cc}
x_{u} & x_{v} \\
y_{u} & y_{v}
\end{array}\right) \equiv x_{u} y_{v}-x_{v} y_{u} ;
$$

throughout, the subscripts $u, v, x, y, x^{0}$, and $y^{0}$ indicate a derivative, e.g., $x_{u}$ denotes $\partial x / \partial u$.

We integrate (15) along any curve $S S^{\prime}$ in Fig. 1, first expanding $g J$ there along that curve, as in the one-dimensional analog in Part II and as in Ref. 4. The leading term is ${ }^{6}$

$$
g J=p_{0}+q_{0} u,
$$

where $p_{0}$ and $q_{0}$ both depend on the $S S^{\prime}$ curve. [In an integration along an $S S^{\prime}$ curve, $u$ varies and $v$ may also. Thus, if $u$ is used as the integration variable along $S S^{\prime}$, any $v$ in the integrand becomes a $\left.v(u).\right]$ At the points $S$ and $S^{\prime}$ in Fig. 1, $u$ has the values in Eq. (7). Upon evaluation of $p_{0}$ and $q_{0}, \mathrm{Eq}$. (17) yields

$$
g J=\frac{1}{2}\left(1-u \zeta^{-1 / 2}\right)(g J)_{S}+\frac{1}{2}\left(1+u \zeta^{-1 / 2}\right)(g J)_{S^{\prime}},
$$

where $(g J)_{S}$ denotes the value of $g J$ at $S$.

We denote by the symbols $a i^{( \pm)}$the following integrals:

$$
\begin{aligned}
& a i^{( \pm)}(-\zeta)=(2 \pi)^{-1} \int_{-\infty}^{\infty}\left(1 \mp u \zeta^{-1 / 2}\right) \\
& \times\left[\exp i\left(\frac{1}{3} u^{3}-\zeta u\right)\right] d u .
\end{aligned}
$$

From the definitions ${ }^{9}$ of the Airy function $A i$ and its derivative, $A i^{\prime}$, we have

$$
a i^{( \pm)}(-\zeta)=A i(-\zeta) \pm i \zeta^{-1 / 2} A i^{\prime}(-\zeta)
$$

Bearing in mind the asymptotic behavior of $Q i^{( \pm)}(-\zeta),{ }^{9}$ it is convenient to introduce a function $B i^{(t)}(-\zeta)$ which tends to unity at large $\zeta$ :

$$
\begin{aligned}
& B i^{( \pm)}(-\zeta)=\pi^{1 / 2} \zeta^{1 / 4} Q i^{( \pm)}(-\zeta) \\
& \times \exp \left[\mp i\left(\frac{2}{3} \zeta^{3 / 2}-\frac{1}{4} \pi\right)\right] .
\end{aligned}
$$

Values of the magnitude and phase of $B i^{(+)}(-\zeta)$ are given for various $\zeta$ 's in the range 0.01 to 3 in Table I. (The value of $B i^{( \pm)}$diverges when $\zeta$ is zero.) Its

FIg. 1. Curvilinear coordinates, $u$ and $v$, and points of stationary phase, $P_{i} . S$ and $S^{\prime}$ are points in which $f^{\prime}(u)=0$ along a $u$ curve.
TABLE I. Amplitude $(\rho)$ and phase $(\phi)$ of $B i^{(+)}(-\zeta)$.

\begin{tabular}{cccccc}
\hline \hline$\zeta$ & $\rho$ & $\phi$ & $\zeta$ & $\rho$ & $\phi$ \\
\hline 0.01 & 1.464 & -0.649 & 0.90 & 0.925 & +0.096 \\
0.03 & 1.134 & -0.551 & 1.0 & 0.949 & +0.100 \\
0.05 & 1.016 & -0.485 & 1.2 & 0.993 & +0.093 \\
0.07 & 0.929 & -0.409 & 1.4 & 1.025 & +0.072 \\
0.10 & 0.894 & -0.368 & 1.6 & 1.044 & +0.044 \\
0.20 & 0.821 & -0.219 & 1.8 & 1.048 & +0.015 \\
0.30 & 0.807 & -0.119 & 2.0 & 1.039 & -0.009 \\
0.40 & 0.813 & -0.048 & 2.2 & 1.020 & -0.024 \\
0.50 & 0.829 & +0.004 & 2.4 & 0.999 & -0.027 \\
0.60 & 0.851 & +0.042 & 2.6 & 0.982 & -0.017 \\
0.70 & 0.874 & +0.069 & 2.8 & 0.975 & -0.002 \\
0.80 & 0.900 & +0.086 & 3.0 & 0.980 & +0.016 \\
\hline \hline
\end{tabular}

magnitude is seen to be fairly close to unity in the region 0.1 to 3 , and its phase changes from -0.37 at $\zeta=0.1$ to values of 0.04 or less when $\zeta$ exceeds 1.6. Ultimately, the phase becomes zero and the magnitude becomes unity at large $\zeta . B i^{(-)}(-\zeta)$ is the complex conjugate of $B i^{(+)}(-\zeta)$.

The integral in Eq. (15) can now be written as

$$
\begin{aligned}
& I=\pi^{1 / 2} \int \zeta^{-1 / 4}\left\{(g J)_{S}\left[\exp i\left(\frac{2}{3} \zeta_{S S^{3}}{ }^{3 / 2}+A-\frac{1}{4} \pi\right)\right]\right. \\
& \times B i^{(+)}\left(-\zeta S S^{\prime}\right)+(g J)_{S^{\prime}}\left[\exp i\left(-\frac{2}{3} \zeta_{S S^{\prime}}{ }^{3 / 2}+A+\frac{1}{4} \pi\right)\right] \\
& \left.\times B i^{(-)}\left(-\zeta_{s s^{\prime}}\right)\right\} d v \text {. }
\end{aligned}
$$

Introducing (8a) into (20) and noting that $S$ lies on curve $P_{1} P_{2}$ and that $S^{\prime}$ lies on curve $P_{4} P_{3}$, Eq. (20) may be rewritten as

$$
\begin{aligned}
I= & \pi^{1 / 2} \int_{P_{1} P_{2}} \zeta_{S S^{\prime}}{ }^{-1 / 4} g J B i^{(+)}\left(-\zeta_{S S^{\prime}}\right) \exp i\left(f-\frac{1}{4} \pi\right) d v \\
& +\int_{P_{4} P_{3}} \zeta_{S S^{\prime}}-1 / 4 g J B i^{(-)}\left(-\zeta_{S S^{\prime}}\right) \exp i\left(f+\frac{1}{4} \pi\right) d v .
\end{aligned}
$$

In integrating along $v$ curves $P_{1} P_{2}$ and $P_{4} P_{3}$ one may use $v$ as an integration variable.

When $\zeta$ is large, $B i^{( \pm)}(-\zeta)$ is about unity, and so the only significant variation in phase of the integrands of (19) is due to $f(x, y)$. However, even more generally, (14a) shows that $d \zeta_{S s^{\prime}} / d v$ vanishes at a stationary phase point and so $\beta i^{( \pm)}\left(\zeta_{S S^{\prime}}\right)$ makes no contribution to phase variation in the integrand at such a point. One can thus treat the factors $\zeta^{-1 / 2} g J B i^{( \pm)}$as in (17), whence we may set

$$
\begin{aligned}
\zeta_{S S^{\prime}}-1 / 4 g J\left(B i^{( \pm)}(\right. & \left.-\zeta_{S S^{\prime}}\right)=P_{0}+Q_{0} v \\
& \left(\text { on } P_{1} P_{2} \text { and } P_{4} P_{3}\right),
\end{aligned}
$$

and evaluate $P_{0}$ and $Q_{0}$. The $(+)$ sign in $B i$ is for $P_{1} P_{2}$ and the (-) sign is for $P_{4} P_{3}$, according to (21), and the values of $P_{0}$ and $Q_{0}$ on the first curve differ from those on the second. $\zeta_{S S^{\prime}}$ is $\zeta_{14}$ at $P_{1}$ and $\zeta_{23}$ at $P_{2}$ and according to (1), $v$ is $-\eta_{12}$ at $P_{1}$ and $+\eta_{12}$ at $P_{2}$. Denoting by $g_{i}$ and $J_{i}$ the values of $g$ and $J$ at point $P_{i}$, 
and introducing these results into (22) to obtain $P_{0}$ and $Q_{0}$, one finds that on curve $P_{1} P_{2}$

$$
\begin{aligned}
\zeta^{-1 / 4} g J \otimes i^{(+)} & (-\zeta) \\
= & \frac{1}{2} \zeta_{14^{-1 / 4}} g_{1} J_{1}\left(1-v \eta_{12}{ }^{-1 / 2}\right) B i^{(+)}\left(-\zeta_{14}\right) \\
& +\frac{1}{2} \zeta_{23}{ }^{-1 / 4} g_{2} J_{2}\left(1+v \eta_{12}{ }^{-1 / 2}\right) B i^{(+)}\left(-\zeta_{23}\right) .
\end{aligned}
$$

Similarly, using (14) and (22), one finds that on curve $P_{4} P_{3}$

$$
\begin{aligned}
\zeta^{-1 / 4} g J\left(B i^{(-)}\right. & (-\zeta) \\
= & \frac{1}{2} \zeta_{14}{ }^{-1 / 4} g_{4} J_{4}\left(1-v \eta_{43}{ }^{-1 / 2}\right) \otimes i^{(-)}\left(-\zeta_{14}\right) \\
& +\frac{1}{2} \zeta_{23}{ }^{-1 / 4} g_{3} J_{3}\left(1+v \eta_{43}{ }^{-1 / 2}\right) B i^{(-)}\left(-\zeta_{23}\right) .
\end{aligned}
$$

We may also write the $f(x, y)$ in (21) as in (9), introduce (23) and (24) into (21), and use (19c) to write the resulting $Q i^{( \pm)}$integrals in terms of $B i^{( \pm)}$'s. When Eqs. (9) and (11) are used to simplify the resulting expression one obtains

$$
\begin{gathered}
I=\left[\pi g_{1} J_{1} /\left(\eta_{12} \zeta_{14}\right)^{1 / 4}\right] \mathrm{B} i^{(+)}\left(-\eta_{12}\right) B i^{(+)}\left(-\zeta_{14}\right) \\
\times \exp i\left(f_{1}-\frac{1}{2} \pi\right)+\left[\pi g_{2} J_{2} /\left(\eta_{12} \zeta_{23}\right)^{1 / 4}\right] \\
\quad \times B i^{(-)}\left(-\eta_{12}\right) B i^{(+)}\left(-\zeta_{23}\right) \exp i f_{2} \\
+\left[\pi g_{3} J_{3} /\left(\eta_{43} \zeta_{23}\right)^{1 / 4}\right] \mathrm{Bi} i^{(-)}\left(-\zeta_{23}\right) B i^{(-)}\left(-\eta_{43}\right) \\
\times \exp i\left(f_{3}+\frac{1}{2} \pi\right)+\left[\pi g_{4} J_{4} /\left(\eta_{43} \zeta_{14}\right)^{1 / 4}\right] \\
\times B i^{(-)}\left(-\zeta_{14}\right) B i^{(+)}\left(-\eta_{43}\right) \exp i f_{4} .
\end{gathered}
$$

This expression can be further simplified: The $J_{i}$ 's can be expressed in terms of properties of $f(x, y)$ and so freed from those of $u$ and $v$. We first note that one can readily show that the second derivatives of $f$ with respect to $x$ and $y$ are related to those with respect to $u$ and $v$. At a point of stationary phase one obtains the first half of (26):

$$
\begin{aligned}
&\left(\begin{array}{cc}
x_{u} & y_{u} \\
x_{v} & y_{v}
\end{array}\right)\left(\begin{array}{ll}
f_{x x} & f_{x y} \\
f_{x y} & f_{y v}
\end{array}\right)\left(\begin{array}{ll}
x_{u} & x_{v} \\
y_{u} & y_{v}
\end{array}\right) \\
&=\left(\begin{array}{cc}
f_{u u} & f_{u v} \\
f_{u v} & f_{v v}
\end{array}\right)=\left(\begin{array}{ll}
2 u & 0 \\
0 & 2 v
\end{array}\right) .
\end{aligned}
$$

The second half of (26) is obtained by differentiating (4) and (9) with respect to $u$ and $v$ at a stationary phase point and using (14) (Appendix B).

The square matrices in (26) can be interpreted as signed determinants. The first factor on the left hand side then equals the third, which in turn is $J$, according to (16). Thus, (26) yields

where

$$
J=2 F^{-1 / 2}(u v)^{1 / 2}
$$

$$
F=\left(\begin{array}{cc}
f_{x x} & f_{x y} \\
f_{x y} & f_{y y}
\end{array}\right)
$$

and the quantities are to be evaluated at the cited point of stationary phase.
Further, the quantity $g$ in Eq. (3) involves a (signed) determinant of derivatives the values of $x$ and $y$ with respect to initial values before the collision, $x^{0}$ and $y^{0}{ }^{1}$ :

$$
g=\left(\begin{array}{cc}
x_{x^{0}} & x_{y^{0}} \\
y_{x^{0}} & y_{y^{0}}
\end{array}\right)^{-1 / 2} .
$$

Equations (27) to (29) yield

where

$$
g J=2 G^{-1 / 2}(u v)^{1 / 2},
$$

$G^{-1 / 2}=g F^{-1 / 2}=\left(\begin{array}{cc}f_{x x^{0}} & f_{x y^{0}} \\ f_{y x^{0}} & f_{y y^{0}}\end{array}\right)^{-1 / 2}=\left(\begin{array}{cc}X_{x^{0}} & X_{y^{0}} \\ Y_{x^{0}} & Y_{y^{0}}\end{array}\right)^{-1 / 2}$.

Here, $X$ denotes $f_{x}$ and $Y$ denotes $f_{y} ; X$ and $Y$ are the final momenta conjugate to the final coordinates $x$ and $y$, respectively. 1,8

When the values of $(u v)^{1 / 2}$ appropriate to the given stationary phase points, namely those given by (13), are introduced, Eq. (25) becomes

$$
\begin{aligned}
I=2 \pi & \exp \left(i \frac{1}{2} \pi\right) \\
& \times\left[G_{1}^{-1 / 2} B i^{(+)}\left(-\eta_{12}\right) B i^{(+)}\left(-\zeta_{14}\right) \exp \left(i f_{1}\right)\right. \\
& +G_{2}^{-1 / 2} B i^{(-)}\left(-\eta_{12}\right) B i^{(+)}\left(-\zeta_{23}\right) \exp \left(i f_{2}\right) \\
& +G_{3}^{-1 / 2} B i^{(-)}\left(-\zeta_{23}\right) B i^{(-)}\left(-\eta_{43}\right) \exp \left(i f_{3}\right) \\
& \left.+G_{4}{ }^{-1 / 2} B i^{(-)}\left(-\zeta_{14}\right) B i^{(+)}\left(-\eta_{43}\right) \exp \left(i f_{4}\right)\right] .
\end{aligned}
$$

We now introduce a completely symmetrical notation for all $i$ and $j$ :

where, therefore, $f_{i}-f_{j}=\frac{4}{3} \zeta_{i j}{ }^{3 / 2}$,

$$
\zeta_{i j}{ }^{3 / 2}=-\zeta_{j i}{ }^{3 / 2} .
$$

From these relations and from the properties of the Airy functions, it can be shown that

$$
B i^{(+)}\left(-\zeta_{i j}\right)=B i^{(-)}\left(-\zeta_{j i}\right) .
$$

Equation (32) now yields

$$
\begin{aligned}
I=2 \pi i \sum_{j=1}^{4} G_{j}^{-1 / 2} \beta i^{(+)}( & \left.-\zeta_{j j_{1}}\right) \\
& \times B i^{(+)}\left(-\zeta_{j j_{2}}\right) \exp \left(i f_{j}\right),
\end{aligned}
$$

where the sum is over all stationary phase points $j$ and where $j_{1}$ and $j_{2}$ denote their adjacent neighbors (i.e., the points on the adjacent corners of the deformed "square" $P_{1} P_{2} P_{3} P_{4}$ in Fig. 1).

When the above type of derivation is used for the case of a one-dimensional integral, $\int g \exp (i f) d x$, one obtains instead

$$
I=(2 \pi i)^{1 / 2} \sum_{j=1}^{2} G_{j}^{-1 / 2}\left(B i^{(+)}\left(-\zeta_{j j^{\prime}}\right) \exp \left(i f_{j}\right),\right.
$$

there being only two stationary phase points in most occasions ( $j^{\prime}=2$ when $j=1$, etc.). 
The generalization of (36) and (37) to $N$ dimensions is apparent from their highly symmetrical form, and is obtained by a straightforward extension of the above arguments. In the case of three dimensions, a three dimensional set of curvilinear coordinates $(u, v, w)$ is introduced, together with the eight points $P_{i}$, and the curves $P_{i j}(i, j=1$ to 8$)$, as in Fig. 2 .

For the $N$-dimensional case one establishes [by induction, since the $N$-dimensional case is the "Cartesian product" of the $(N-1)$ and one dimensional cases] the following result

$$
\begin{array}{r}
I=(2 \pi i)^{N / 2} \sum_{j} G_{j}^{-1 / 2} B i^{(+)}\left(-\zeta_{j j_{1}}\right) \cdots \\
\times \operatorname{Bi} i^{(+)}\left(-\zeta_{j j_{N}}\right) \\
\times \exp \left(i f_{j}\right) .
\end{array}
$$

Since the stationary phase value is given by (38) but with all $B i^{(+)}$'s equal to unity, one can rewrite (38) in terms of the stationary phase contribution from the $j$ 'th point, $I_{j}$ :

$$
I=\sum_{j=1}^{2^{N}} I_{j} B i^{(+)}\left(-\zeta_{j j_{1}}\right) \cdots B i^{(+)}\left(-\zeta_{j j_{N}}\right),
$$

where

$$
I_{j}=(2 \pi i)^{N / 2} G_{j}^{-1 / 2} \text { expif }{ }_{j} .
$$

There are in the sum (39) $2^{N}$ stationary phase points for the system examined, these being the number of corners of an $N$-dimensional hypercube.

\section{DISCUSSION}

When $B i^{(+)}$is written in terms of the Airy integral and its derivative, and when $G$ is expressed in terms of $g$ and $F$, it can be shown (Appendix A) that Eq. (37) leads to the results previously obtained in Part II for one dimension, namely Eqs. (3.20) and (3.26) there.

Again, when $B i^{(+)}$is expressed as above and Eq. (38) then specialized to the case of two dimensions it yields an equation of Miller $^{20}$ for the two-dimensional case, apart from one difference noted earlier ${ }^{2 \mathrm{~b}}: B i(-\zeta)$, the irregular Airy function appears in Ref. 2 instead of the present $\zeta^{-1 / 2} A i^{\prime}(-\zeta)$. The difference arises from the fact that in Ref. 2 the uniform approximation was partly deduced from an interpolation of stationary phase values, and that both $\zeta^{-1 / 2} A i^{\prime}(-\zeta)$ and $B i(-\zeta)$ have the same limiting values, and partly from a result of Carrier, ${ }^{7,10}$ which in turn omitted the second term in (15). The difference in the two functions is, as already mentioned in Part II, negligible when $\zeta$ is large, their limiting behavior being the same. When $\zeta$ is small, they do differ appreciably, but then the term which contains them is largely dominated by one which contains $A i(-\zeta)$.

Equation (39) has, because of its symmetry, several useful properties. It shows immediately that $I$ equals its stationary phase value $\sum_{j} I_{j}$ when the $\zeta$ 's are large, i.e., when the points of stationary phase are widely separated, since the $B i^{(+)}(-\zeta)$ 's approach unity at large $\zeta$. Again, when $\zeta$ is not large but not so small that
Frg. 2. Curvilinear coordinates, $u, v$, and $w$ and points of stationary phase, $P_{i}$.

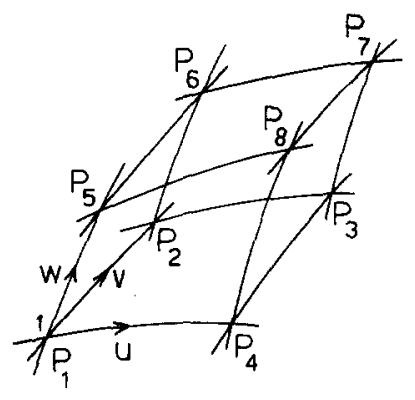

$B i^{(+)}$becomes singular (we note, however, that because of cancellations, $I$ itself is not singular), one can understand from (39) the interesting empirical observation ${ }^{20}$ that the quantum value for $\left|S_{m n}\right|^{2}$ is sometimes closer to the "classical value", $\sum_{j}\left|I_{j}\right|^{2}$ than it is to the stationary phase value $\left|\sum_{j} I_{j}\right|^{2}$ : Some randomness in the $\zeta$ 's in (39) leads to some randomness in the phases of the $B i^{(+)}$'s, leading to $|I|^{2}$ being closer, on such occasions, to $\sum_{j}\left|I_{j}\right|^{2}$. This value, $\sum_{j}\left|I_{j}\right|^{2}$, which we have called classical, was derived earlier in Part I of this series by classical arguments using slightly different notation [see Eq. (2.3) and subsequent sentence there].

To evaluate the phase of $G$ in (34)-(38), one needs some examination of the topography of the $f$ surface, and we consider it now. ${ }^{11}$

In the case of one dimension the stationary phase point at $u=-\zeta^{1 / 2}$ has a negative value of $f_{x x}$ in the classically accessible case, since $f_{x x}$ equals $2 u(d u / d x)^{2}$ there. Since $f_{x x}<0$ at this point (we will call it $P_{1}$ ) $f$ is a maximum there, while at the other point $\left(P_{2}\right) f$ is a minimum. (Indeed, $f_{1}-f_{2}$ equals $\frac{4}{3} \zeta^{3 / 2}$ and is positive.) In (37) one has, $\operatorname{denoting} f_{x x}$ by $f^{\prime \prime}$,

$$
F_{1}=f_{1}^{\prime \prime}=\left|f_{1}^{\prime \prime}\right| \exp i \pi, \quad F_{2}=f_{2}^{\prime \prime}=\left|f_{2}^{\prime \prime}\right| \text {. }
$$

In the case of two dimensions, as in Fig. 1, there is a maximum and a minimum in $f$ along each of the curves $P_{i} P_{j}$. Thereby, at one of the four points $\left(P_{1}\right.$, when $\zeta_{12}$ and $\zeta_{14}$ are positive) $f$ is a maximum, while at the opposite corner, $P_{3}, f$ is a minimum. At each of the other points, $P_{2}$ and $P_{4}$, the $f$ surface has a col. Thereby, the phase of $F^{-1 / 2}$ is given by (42):

$$
\begin{aligned}
& F_{1^{-1 / 2}}=\left|F_{1}\right|^{-1 / 2} \exp (-i \pi), \\
& F_{2}^{-1 / 2}=\left|F_{2}\right|^{-1 / 2} \exp (-i \pi / 2), \\
& F_{3}^{-1 / 2}=\left|F_{3}\right|^{-1 / 2}, \\
& F_{4}^{-1 / 2}=\left|F_{4}\right|^{-1 / 2} \exp (-i \pi / 2),
\end{aligned}
$$

(e.g., when the matrix $F$ in (28) is diagonalized in the neighborhood of a point of stationary phase of $f$, there are two negative values for the diagonal elements at $P_{1}$, leading to a phase of $2 \pi$ for $F_{1}$ and hence to $-\pi$ for $F_{1}^{-1 / 2}$. Related remarks apply to the other $F_{i}$ 's).

In the case of three dimensions, we may consider eight points $P_{1}, \cdots, P_{8}$, as in Fig. 2, with coordinates 
$(u, v, w):$

$$
\begin{aligned}
& P_{1}\left(-\zeta_{14^{1 / 2}},-\zeta_{12}{ }^{1 / 2},-\zeta_{15}{ }^{1 / 2}\right) \text {, } \\
& P_{2}\left(-\zeta_{23}{ }^{1 / 2},+\zeta_{12}{ }^{1 / 2},-\zeta_{26}{ }^{1 / 2}\right) \text {, } \\
& P_{3}\left(+\zeta_{23}{ }^{1 / 2},+\zeta_{43}^{1 / 2},-\zeta_{37^{1 / 2}}\right) \text {, } \\
& P_{4}\left(+\zeta_{14}{ }^{1 / 2},-\zeta_{43^{1 / 2}},-\zeta_{48^{1 / 2}}\right) \text {, } \\
& P_{5}\left(-\zeta_{58}{ }^{1 / 2},-\zeta_{56}^{1 / 2},+\zeta_{15}^{1 / 2}\right) \text {, } \\
& P_{6}\left(-\zeta_{67^{1 / 2}},+\zeta_{56}{ }^{1 / 2},+\zeta_{26}{ }^{1 / 2}\right) \text {, } \\
& P_{7}\left(+\zeta_{67^{1 / 2}},+\zeta_{87^{1 / 2}},+\zeta_{37^{1 / 2}}\right) \text {, } \\
& P_{8}\left(+\zeta_{58}{ }^{1 / 2},-\zeta_{87^{1 / 2}},+\zeta_{48^{1 / 2}}\right) \text {, }
\end{aligned}
$$

where all the $\zeta$ 's cited in (43) are positive. One can always define the $(u, v, w)$ coordinates so that this condition is fulfilled.

From (43) one can see that $P_{1}$ is the point where the $f$ surface has a maximum. At diagonally opposite point $P_{7} f$ has a minimum. At the other points there are various types of saddle points, those adjacent to $P_{1}$, namely $P_{2}, P_{4}$, and $P_{5}$, having maxima in two principal directions and a minimum in a third, while those adjacent to $P_{7}\left(P_{3}, P_{6}, P_{8}\right)$ have minima in two principal directions and a maximum in the third. One can then deduce that

$$
\begin{aligned}
& F_{1}^{-1 / 2}=\left|F_{1}\right|^{-1 / 2} \exp (-3 i \pi / 2), \\
& F_{2}^{-1 / 2}=\left|F_{2}\right|^{-1 / 2} \exp (-i \pi), \\
& F_{3}^{-1 / 2}=\left|F_{3}\right|^{-1 / 2} \exp (-i \pi / 2), \\
& F_{4}^{-1 / 2}=\left|F_{4}\right|^{-1 / 2} \exp (-i \pi), \\
& F_{5}^{-1 / 2}=\left|F_{5}\right|^{-1 / 2} \exp (-i \pi), \\
& F_{6}^{-1 / 2}=\left|F_{6}\right|^{-1 / 2} \exp (-i \pi / 2), \\
& F_{7}^{-1 / 2}=\left|F_{7}\right|^{-1 / 2}, \\
& F_{8}^{-1 / 2}=\left|F_{8}\right|^{-1 / 2} \exp (-i \pi / 2),
\end{aligned}
$$

in the case of classically accessible cases. Once again, these results follow from (43) by noting that a minus sign before a $\zeta^{1 / 2}$ indicates that $f$ is locally a maximum along that coordinate, while a plus sign indicates that it is locally a minimum. The phase of $F_{i}$ equals $\pi$ times the number of such minus signs.

The phase of $G_{i}^{-1 / 2}$ is the same as that of $F_{i}^{-1 / 2}$ when the phase of (29) is zero. In the actual numerical cases examined thus far in the rotation-translation and vibration-translation collisions problems, the phase of (29) has been zero at the points of stationary phase, although it has sometimes differed from zero elsewhere. ${ }^{12}$ Thus, for these cases, the phases in (41), (42), and (43) may be used as the phases of $G^{-1 / 2}$. However, strictly speaking, this question regarding the possible change of phase of (29) should be checked in each instance.

In a plot of the final collisional value of the quantum number $\bar{n}_{k}$ for the $k$ th degree of freedom versus $\left(x^{0}, y^{0}\right)$, the local minimum or the maximum $\bar{n}_{i}$ of such a plot may sometimes be near a stationary phase point of $f(x, y)$ and hence, near an $m_{k}$. In this case the stationary phase points of at least one pair approach each other and eventually coalesce when $m_{k}$ equals the local maximum or minimum $\bar{n}_{k}$. The pair then move in opposite directions along an imaginary axis in a complex $(u, v)$ or $(u, v, \cdots)$ plane. In this case, as in the one-dimensional analog in Part II, the $g$ 's at these two points are complex conjugates, as are the $F$ 's. The associated $\zeta_{i j}$ value is still real but negative. With care, the change in phase from the values given in (41), (42), and (44) can be determined by analytic continuation arguments. Equation (39) remains valid for this classically inaccessible case.

Examination of Eq. (13) shows that if some $P_{i}{ }^{\prime}$ s are complex-valued, the $\zeta_{i j}$ 's can also be complex. Since real $\zeta_{i j}$ 's were assumed originally, one should now modify the argument. Elsewhere, it will be shown how to modify Eq. (38) for such a case.

\section{ACKNOWLEDGMENT}

Dr. Holger Kreek of this laboratory kindly computed the data in Table I and I am pleased to acknowledge his help in this matter, and helpful discussions with Dr. Kreek and Dr. J. N. L. Connor.

\section{APPENDIX A: COMPARISONS}

One can readily show that Eq. (37) is equivalent to Eqs. (3.20) and (3.26) of Part II: according to (31) $G^{-1 / 2}$ equals $g F^{-1 / 2}$, and so (denoting $f_{x x}$ by $f^{\prime \prime}$ ) we have

$$
\begin{aligned}
I=(2 \pi i)^{1 / 2}\left[g_{1}\left(f_{1}^{\prime \prime}\right)^{-1 / 2}\left(\exp i f_{1}\right) B i i^{(+)}\left(-\zeta_{12}\right)\right. \\
\left.+g_{2}\left(f_{2}^{\prime \prime}\right)^{-1 / 2}\left(\exp i f_{2}\right) B i^{(+)}\left(-\zeta_{21}\right)\right] .
\end{aligned}
$$

In (3.20) one had $f_{1}{ }^{\prime \prime}>0$ and ${f_{2}}^{\prime \prime}<0$ for a classically accessible transition, so that $f$ is a minimum at $P_{1}$ and a maximum at $P_{2}$. Since $\frac{4}{3} \zeta_{21}$ equals $f_{2}-f_{1}, \zeta_{21}{ }^{3 / 2}$ is therefore positive and hence $\zeta_{21}$ is also; $\zeta_{12}$ is not. Setting $B i^{(+)}\left(-\zeta_{12}\right)$ equal to $B i^{(-)}\left(-\zeta_{21}\right)$, as in (35), and introducing (19) one obtains

$$
\begin{gathered}
I=(2 i)^{1 / 2} \pi \zeta^{1 / 4}\left\{g_{1}\left(f_{1}^{\prime \prime}\right)^{-1 / 2}\left(\exp i f_{1}\right)\right. \\
\times\left[A i(-\zeta)-i \zeta^{-1 / 2} A i^{\prime}(-\zeta)\right] \\
\times \exp i\left(\frac{2}{3} \zeta^{3 / 2}-\frac{1}{4} \pi\right)+g_{2}\left(f_{2}^{\prime \prime}\right)^{-1 / 2}\left(\exp i f_{2}\right) \\
\left.\times\left[A i(-\zeta)+i \zeta^{-1 / 2} A i^{\prime}(-\zeta)\right] \exp -i\left(\frac{2}{3} \zeta^{3 / 2}-\frac{1}{4} \pi\right)\right\}
\end{gathered}
$$

where $\zeta$ denotes $\zeta_{21}$.

Since $f^{\prime \prime}$ equals $2 u(d u / d x)^{2}$, point $P_{1}$ must be at $u=+\zeta^{1 / 2}$, to have $f_{1}^{\prime \prime}>0$, and point $P_{2}$ must be at $u=-\zeta^{1 / 2}$. Thus, $f_{1}$ equals $-\frac{2}{3} \zeta^{3 / 2}+A$ and $f_{2}$ equals $\frac{2}{3} \zeta^{3 / 2}+A$, a result which simplifies (A2). Finally, since $f_{1}^{\prime \prime}$ is $\left|f_{1}^{\prime \prime}\right|$ and $f_{2}^{\prime \prime}$ is $\left|f_{2}^{\prime \prime}\right|$ expi $i, f_{2}^{\prime \prime}$ being negative, one can introduce this result and the preceding one into 
(A2) and obtain (3.20) of Part II. When Eq. (3.26) of Part II is regarded as a relabeling of points 1 and 2, by changing the sign of the original coordinates, it does not constitute a separate problem.

\section{APPENDIX B: PROOF OF SECOND HALF OF EQ. (26)}

We first note that the curves labeled for convenience as $u$ and $v$ curves in Fig. 1 are not the usual curvilinear $u$-coordinate and $v$-coordinate curves. For example, on a $u$ curve $P_{1} P_{4}, v$ is given by (11) and so is not in general constant on it. Similarly, on a $v$ curve $P_{1} P_{2}$ the quantity $u$, given by (7), is not in general constant on it. ${ }^{13}$

Along a $u$ curve, such as $P_{1} P_{4}$ or $P_{2} P_{3}$, we have

$$
d f / d u=\partial f / \partial u+(\partial f / \partial v)(d v / d u)
$$

where $d / d u$ will denote differentiation along a $u$ curve, and $d / d v$ will denote differentiation along a $v$ curve. (Thereby, with this notation $d v / d u$ and $d u / d v$ are not reciprocals.)

Differentiation of Eq. (B1) and of Eq. (4) along a $u$ curve shows that

$2 u=\frac{d^{2} f}{d u^{2}}=\frac{\partial^{2} f}{\partial u^{2}}+2 \frac{\partial^{2} f}{\partial u \partial v} \frac{d v}{d u}+\frac{\partial f}{\partial v} \frac{d^{2} v}{d u^{2}}+\frac{\partial^{2} f}{\partial v^{2}}\left(\frac{d v}{d u}\right)^{2}$.

At a stationary phase point, Eqs. (11) and (14b) show that $d v / d u$ vanishes. According to (5) $\partial f / \partial v$ also vanishes there. Thus, (B2) shows that, at a stationary phase point $P_{i}, \partial^{2} f / \partial u^{2}$ equals $2 u$, as in (26). Similarly, $\partial^{2} f / \partial v^{2}$ equals the $d^{2} f / d v^{2}$ computed along a $v$ curve at a $P_{i}$ and so, from (9), equals $2 v$, again as in (26).

Differentiating (B1) along a $v$ curve yields (B3). Differentiating (4) first along a $u$ curve and then along a $v$ curve yields (B4)

$$
\begin{gathered}
\frac{d(d f / d u)}{d v}=\frac{\partial^{2} f}{\partial u \partial v}+\frac{\partial^{2} f}{\partial u^{2}} \frac{d u}{d v}+\frac{\partial f}{\partial v} \frac{d[(d v / d u)]}{d v} \\
+\frac{\partial^{2} f}{\partial v \partial u} \frac{d u}{d v} \frac{d v}{d u}+\frac{\partial^{2} f}{\partial v^{2}} \frac{d v}{d u} \\
\frac{d(d f / d u)}{d v}=2 u \frac{d u}{d v}-\frac{d \xi}{d v} .
\end{gathered}
$$

Since $d v / d u$ vanishes at a $P_{i}$, as already noted, and similarly according to (7) and (14a) $d u / d v$ and $d \zeta / d v$ also vanish at a $P_{i}$, as also does $\partial f / \partial v,(\mathrm{~B} 3)$ and (B4) show that $\partial^{2} f / \partial u \partial v$ vanishes, as in (26).

* Acknowledgement is made to the donors of the Petroleum Research Fund, administered by the American Chemical Society, and to the National Science Foundation for their support of this research.

1 (a) R. A. Marcus, Chem. Phys. Letters 7, 525 (1970); J. Chem. Phys. 54, 3965 (1971) (Part I); (b) J. N. L. Connor and R. A. Marcus, ibid. 55, 5636 (1971) (Part II); (c) W. H. Wong and R. A. Marcus, ibid. 55, 5663 (1971); (d) R. A. Marcus, ibid. 56, 311 (1972) (Part III) ; (e) J. Stine and R. A. Marcus, Chem. Phys. Letters 15, 536 (1972) (Part IV).

2 (a) W. H. Miller, J. Chem. Phys. 53, 1949 (1970); (b) 53, 3578 (1970); (c) 54, 5386 (1971); (d) Chem. Phys. Letters 7, 431 (1970); (e) Accts. Chem. Res. 4, 161 (1971); (f) C. C. Rankin and W. H. Miller, J. Chem. Phys. 55, 3150 (1971).

${ }^{3}$ Other examples of recent semiclassical work include: (a) $R$. D. Levine and B. R. Johnson, Chem. Phys. Letters 7, 404 (1970); 8, 501 (1971); (b) P. Pechukas, Phys. Rev. 181, 166, 174 (1969); (c) I. L. Beigman, L. A. Varnshtein, and I. I. Sobel'man, Zh. Eksp. Teor. Fiz. 57, 1703 (1969); [Sov. Phys. JETP 30, 920 (1970) ]; (d) I. C. Percival and D. Richards, J. Phys. B 3, 315, 1035 (1970).

${ }^{4} \mathrm{C}$. Chester, B. Friedman, and F. Ursell, Proc. Cambridge Phil. Soc. 53, 599 (1957) ; B. Friedman, J. Soc. Ind. Appl. Math. 7, 280 (1959).

${ }_{5}^{5}$ M. V. Berry, Proc. Phys. Soc. 89, 479 (1966) ; J. Phys. B 2, 381 (1969).

${ }^{6}$ In our notation, the pre-exponential factor in Ref. 4 is expanded as a sum,

$$
\sum_{m=0}^{\infty}\left(u^{2}+\zeta\right)^{m}\left(p_{m}+q_{m} u\right)
$$

In Part II [Ref. 2(b)] and in the present paper we use the $m=0$ term.

${ }^{7}$ References 2(b)-2(c). E.g., cf discussion in Appendix $A$ of Ref. 2(b) and in Appendix D of Ref. 2(c) [That method has also been used to treat rainbow and glory scattering. W. $H$. Miller, J. Chem. Phys. 48, 464 (1968).]

${ }^{8}$ In Part $\mathrm{I}, x$ and $y$ angle variables are $w_{i}$ 's. After a canonical transformation in Part III, they are $\bar{w}_{i}$ 's, which are "reduced" (constant in time) angle variables. As noted in Part III, the uniform approximations for the two integrals agree identically.

'M. Abramowitz and I. A. Stegun (Eds.), National. Bur. Std. (U.S.) Appl. Math. Ser. 55, 446 (1964).

${ }^{10} \mathrm{G}$. F. Carrier, J. Fluid Mech. 24, 641 (1966).

11 The phase of the pre-exponential factor has also been discussed in terms of the eigenvalues of a Jacobian, in W. H. Miller, J. Chem. Phys. 54, (1971).

${ }^{12} \mathrm{H}$. Kreek and J. Stine, unpublished results in this laboratory.

${ }^{13} \mathrm{It}$ is perhaps worthwhile remarking here that in Eq. (4) one should not treat $\zeta$ and $A$ as functions of $v$ alone: $\zeta$ and $A$ are constant along any $u$ curve but, as already noted, $v$ can vary along such a curve. Thus, $\zeta$ and $A$ are functions of both $u$ and $v$, in such a way that $d \zeta / d u$ and $d A / d u$ vanish along $u$ curves. Similarly, $\eta$ and $B$ in Eq. (9) can be written as $\eta(u, v)$ and $B(u, v)$, but are constant along $v$ curves. 